\title{
Major and ancillary features according to LI-RADS in the assessment of combined hepatocellular-cholangiocarcinoma
}

\author{
Vincenza Granata ${ }^{1}$, Roberta Fusco ${ }^{1}$, Sergio Venanzio Setola ${ }^{1}$, Fabio Sandomenico \\ Maria Luisa Barrettaํㅡㄹ Andrea Belli², Raffaele Palaia², Fabiana Tatangelo ${ }^{3}$, Roberta Grassi ${ }^{4}$, \\ Francesco $\mathrm{IzzO}^{2}$, Antonella Petrillo

\begin{abstract}
${ }^{1}$ Radiology Division, “Istituto Nazionale Tumori IRCCS Fondazione Pascale - IRCCS di Napoli”, Naples, Italy
${ }^{2}$ Hepatobiliary Surgical Oncology Division, “Istituto Nazionale Tumori IRCCS Fondazione Pascale - IRCCS di Napoli”, Naples, Italy

${ }^{3}$ Pathology Diagnostic Division, “Istituto Nazionale Tumori IRCCS Fondazione Pascale - IRCCS di Napoli”, Naples, Italy

${ }^{4}$ Division of Radiology, University of Campania Luigi Vanvitelli, Naples, Italy
\end{abstract}

Radiol Oncol 2020; 54(2): 149-158.

Received 6 February 2020

Accepted 22 April 2020

Correspondence to: Roberta Fusco, M.D., Department of Radiology, Istituto Nazionale Tumori Fondazione G. Pascale, Naples, I-80131, Italy. E-mail: r.fusco@istitutotumori.na.it

Disclosure: No potential conflicts of interest were disclosed.

Background. The aim of the study was to investigate the performance of the Liver Imaging Reporting and Data System (LI-RADS) v2018 for combined hepatocellular-cholangiocarcinoma (CHCC-CCA) identifying the features that allow an accurate characterization.

Patients and methods. Sixty-two patients (median age, 63 years; range, 38-80 years), with pre-surgical biopsy diagnosis of hepatocellular carcinoma $(\mathrm{HCC})$ that underwent hepatic resection, comprised our retrospective study. All patients were subject to multidetector computed tomography (MDCT); 23 patients underwent to magnetic resonance (MR) study. The radiologist reported the presence of the HCC by using LIRADS v2018 assessing major and ancillary features.

Results. Final histological diagnosis was $\mathrm{HCC}$ for 51 patients and $\mathrm{CHCC}-\mathrm{CCA}$ for 11 patients. The median nodule size was $46.0 \mathrm{~mm}$ (range 10-190 mm). For CHCC-CCA the median size was $33.5 \mathrm{~mm}$ (range 20-80 mm), for true HCC the median size was $47.5 \mathrm{~mm}$ (range 10-190 mm). According to LIRADS categories: 54 (87.1\%) nodules as defined as LR-5, 1 (1.6\%) as LR-3, and 7 (11.3\%) as LR-M. Thirty-nine nodules (63\%) showed hyper-enhancement in arterial phase; among them 4 were CHCC-CCA (36.4\% of CHCC-CCA) and 35 (68.6\%) true HCC. Forty-three nodules (69.3\%) showed washout appearance; 6 cHCC-CCAs $(54.5 \%$ of $\mathrm{CHCC}-\mathrm{CCA}$ ) and 37 true HCC $(72.5 \%)$ had this feature. Only two cHCCCCA patients (18.2\% of CHCC-CCA) showed capsule appearance. Five CHCC-CCA (71.4\% of CHCC-CCA) showed hyperintensity on T2-W sequences while two (28.6\%) showed inhomogeneous signal in T2-W. All CHCC-CCA showed restricted diffusion. Seven CHCC-CCA patients showed a progressive contrast enhancement and satellite nodules.

Conclusions. The presence of satellite nodules, hyperintense signal on $T 2-W$, restricted diffusion, the absence of capsule appearance in nodule that shows peripheral and progressive contrast enhancement are suggestive features of CHCC-CCA.

Key words: hepatocellular carcinoma; combined hepatocellular-cholangiocarcinoma; multidetector computed tomography; magnetic resonance imaging.

\section{Introduction}

Combined hepatocellular-cholangiocarcinoma (cHCC-CCA) is considered a rare entity of primary liver tumour consisting of mixed elements of hepatocellular carcinoma (HCC) and cholangiocarcinoma (CCA) or cancer cells with hepatic progenitor/stem cell traits. ${ }^{1,2}$ The incidence of cHCC-CCA 
ranging from $1.0 \%-4.7 \%$ of all primary hepatic tumours. ${ }^{3,4}$ Described risk factors are male gender, cirrhosis, hepatitis infection (hepatitis B virus $[\mathrm{HBV}]$ and hepatitis $\mathrm{C}$ virus $[\mathrm{HCV}])$, family history of liver cancer, heavy alcohol consumption and diabetes mellitus. ${ }^{5-10}$ Therefore, that cHCC-CCA is associated with overlapping clinical features of both HCC and CCA. ${ }^{3}$ An exact pre-surgical diagnosis is very complicated and, it is due to its heterogeneous imaging characteristics with overlapping features of HCC and CCA. The predominant histologic elements within the tumour determine the predominant radiographic features. ${ }^{3}$ Therefore, in this scenario the hallmark radiological findings of HCC show an overlapping with those of CCA. ${ }^{3}$ Since CHCC-CCAs are predominant in patients at high risk of HCC, and the possibility that this tumour can mimic HCC in imaging appearance, this is problematic considering the current dependence on the non-invasive diagnosis of HCC. ${ }^{11-17}$ Specifically, given that surgical resection is the current standard of care for CHCC-CCAs and controversies surround the appropriateness of other therapies, such us ablative therapies, imaging misdiagnosis of cHCC-CCA can lead to non standard treatments for cHCC-CCA. ${ }^{18}$ The current imaging-based criteria to characterize a HCC lesion have several limitations, including the lack of established consensus regarding the exact definitions of imaging features, binary categorization (either definite or not definite HCC), and failure to address non-HCC malignancies and vascular invasion. ${ }^{13}$ Therefore, The American College of Radiology sustained the spread of Liver Imaging Reporting and Data System (LI-RADS) to homogenizing the interpreting, reporting and data collection of HCC imaging. LI-RADS is a scheme for interpreting and reporting of imaging features on multidetector computed tomography (MDCT) and magnetic resonance (MR) studies in patients at risk for HCC. In the current (v2018) LI- RADS ${ }^{19}$, the diagnosis of HCC is based on the presence of major imaging features. These are features used to categorize LI-RADS-category 3 (LR-3), LI-RADS- category 4 (LR-4), and LI-RADScategory 5 (LR-5) and include arterial-phase hyperenhancement, tumour diameter, washout appearance, capsule appearance, and thresh-old growth. Ancillary features favoring HCC diagnosis include the hepatobiliary phase hypointensity (after administration of liver-specific MR contrast agent), transitional phase hypointensity, mild to moderate T2 hyperintensity, restricted diffusion, distinctive rim, corona enhancement, mosaic architecture, nodule-in-nodule architecture, intra-lesional fat, lesional iron or fat sparing, blood products, and diameter increase less than the threshold growth. The presence of ancillary features favoring malignancy may be used to up- grade by one category, but not beyond LR-4 (e.g. from LR-3 to LR-4). Absence of ancillary features must not be used to downgrade an LR category. ${ }^{19}$

The purpose of this study is to investigate the performance of the LI-RADS v2018 for cHCC-CCA identifying which features allow an exact characterization respected to HCC.

\section{Patients and methods}

\section{Study population}

The institutional review board approved this retrospective study, and the requirement for patient informed consent was waived. We searched the surgical database at our institution from January 2013 to September 2018 and selected 74 patients with pre surgical biopsy and radiological diagnosis of HCC, who underwent hepatic resection. The inclusion criteria for the study population were as follows: (a) patients who had pathologicallyproven HCC; (b) patients who had undergone MR imaging and liver MDCT with less than a 1-month interval between imaging modalities; (c) patients who had less than a 1-month interval between imaging and pathologic diagnosis; and (d) availability of diagnostic quality pictures of the cut sections of the resected specimens in patients who underwent surgical resection for matching of imaging and pathology findings. The exclusion criteria were as follows: (a) conflict between the imaging-based diagnosis and the pathologically confirmed diagnosis, (b) no available MR or MDCT images.

In total, 73 patients with HCC confirmed at pathology fulfilled the inclusion criteria during the study period. Among them, 11 patients were excluded for the following reasons: (a) 4 patients no had available MR or MDCT images and (b) 7 patients because the final diagnosis were not HCC. Finally, 62 patients (14 women, 48 men; median age, 63 years; range, $38-80$ years), with pre-surgical biopsy and radiological diagnosis of HCC comprised our study population. Characteristics of the 62 patients are summarized in Table 1.

\section{Lesion confirmation: reference standard}

All original pathological samples were reviewed by one experienced hepatic pathologist (F.T.). Lesions were confirmed histopathologically as he- 
patic tumours comprising unequivocal elements of both HCC and CC according to the tumour classification of the World Health Organization. The CC component was defined as glandular differentiation with mucin production, while the HCC component was defined as trabecular, solid sheet, or pseudoacinar arrangements with interspersed sinusoids. All pathological samples displayed an intimate intermingling of trabecular hepatocellular and true glandular elements (type C). The bidirectional differentiation was further supported by immunohistochemical stain. For each specimen, biliary differentiation was confirmed with mucin positivity or with immunohistochemical stains characteristic of bile duct differentiation (cytokeratin 7, cytokeratin 19), whereas hepatocellular differentiation was confirmed with immunohistochemical stains characteristic of hepatocyte differentiation (Hepatocyte-Paraffin-1).

\section{MDCT and MR examinations}

All patients underwent to MDCT and 23 to MR.

\section{MDCT protocol}

MDCT was performed with a 64-detector row scanner (Optima 660, GE Healthcare, United States). MDCT scanning parameters were $120 \mathrm{kVp}, 100$ $470 \mathrm{mAs}$ (NI 16.36), $2.5 \mathrm{~mm}$ slice thickness and table speed $0.984 / 1 \mathrm{~mm} /$ rotation. Scans were carried out including a region encompassing the liver from diaphragm to iliac crests. Liver protocol examinations were composed of quadruple phases, including the unenhanced, arterial, portal venous, and equilibrium phases. CT images were obtained after injection of $120 \mathrm{~mL}$ of a nonionic contrast medium (iomeprol, Iomeron 400, Bracco, Milan, Italy) at a rate of $3.0-4.0 \mathrm{~mL} / \mathrm{sec}$ by using an automatic power injector (Empower CTA, E-Z-EM Inc., New York, United States). Image acquisition in the arterial phase was initiated 19 seconds after attenuation in the descending aorta reached $100 \mathrm{HU}$, as measured with the bolus tracking method; in the portal venous phase, images were acquired 33 seconds after the arterial phase; in the equilibrium phase, images were acquired 180 seconds after administration of contrast media.

\section{MR imaging protocol}

MR imaging was performed by using a $1.5 \mathrm{~T}$ scanner (Magnetom Symphony, with Total Imaging Matrix Package, Siemens, Erlangen, Germany) with
TABLE 1. Characteristics of the 62 selected patients

\begin{tabular}{lc}
\hline Description & Numbers (\%)/ range \\
\hline Gender & Men 48 (77.4\%) \\
Age & 63 y; range. 38-80 y \\
Number of hepatic nodules & \\
Single nodule & 62 (100\%) \\
Multiple nodules & $/$ \\
Nodule size (mm) & median size $46.0 \mathrm{~mm} ;$ \\
Risk factor for HCC & range 10-190 mm \\
Chronic hepatitis B; HBV-related liver cirrhosis & 37 (59.7\%) \\
Chronic hepatitis C; HCV-related liver cirrhosis & $23(37.1)$ \\
Alcoholic liver cirrhosis & 2 (3.2\%) \\
Child-Pugh Classification & 62 (100\%) \\
A & \\
B &
\end{tabular}

$\mathrm{HBV}=$ hepatitis $\mathrm{B}$ virus; $\mathrm{HCC}=$ hepatocellular carcinoma; hepatitis $\mathrm{C}$ virus

an 8-element body coil and a phased array coil. Our routine liver MR imaging protocol consisted of a breath-hold fat-saturated and not fat-saturated T2weighted turbo spin-echo sequence, an in- and opposed-phase T1-weighted gradient-echo sequence, dynamic imaging with a fat-saturated T1-weighted gradient-echo sequence, and diffusion-weighted imaging. Diffusion weighted imaging (DWI) was obtained with planar echo-pulse sequence ( $b$ values $0,50,100,200,400,600$, and $\left.800 \mathrm{~s} / \mathrm{mm}^{2}\right)$. A non-specific agent the Gd-BT-DO3A (Gadovist, Bayer Schering Pharma, Germany) was employed. All patients received $0.1 \mathrm{ml} / \mathrm{kg}$ of Gd-BT-DO3A by means of a power injector (Spectris Solaris ${ }^{\circledR}$ EP MR, MEDRAD Inc., Indianola, IA, USA), at an infusion rate of $2 \mathrm{ml} / \mathrm{s}$ followed by a 30-mL saline flush. Arterial phase images were acquired 7 seconds after contrast material arrival at the thoracic aorta by using an MR fluoroscopic monitoring system. Thereafter, portal venous phase and equilibrium phase were obtained 60 seconds and 3 minutes after contrast material administration, respectively. Detailed information regarding the MR imaging parameters are summarized in Table 2.

\section{Image analysis}

For each patient, MDCT and MR images were independently and blindly evaluated in random or- 
der within and between three radiologists (V.G., S.V.S., A.P.; 10, 15, and 20 years of experience in abdominal imaging). A consensus evaluation was performed when there was disagreement between the readers. The readers were blinded to previous radiological examination, pathologic results and history of previous treatment but were aware that the patients had cirrhosis and thus were at higher risk for HCC. To reduce recall bias, all three readers maintained an interval of more than 2 weeks between interpretation sessions of MR and MDCT images.

Each radiologist was asked to identify the presence of lesion, that was considered to be detectable if the nodule had attenuation or signal intensity that differed from that of the surrounding liver parenchyma. Thereafter, they reported the presence of the HCC by using LIRADS v2018 assessing major and ancillary features ${ }^{19}$; also the radiologists reported any radiological accessory findings if detected.

Readers assessed and recorded the following parameters: greatest nodule diameter, attenuation at unenhanced $\mathrm{CT}$, signal intensity on T1- and T2-weighted images, vascular hyperenhancement pattern during arterial phase (wash-in), wash-out appearance during portal phase, vascular enhancement during equilibrium or late phases. ${ }^{13}$

Region of interests (ROIs) have been manually drawn by an expert radiologist on T1-w and T2-w images and on DW images at the highest $b$ value (including hyperintense voxels at $\mathrm{b}$ value $800 \mathrm{~s} /$ $\mathrm{mm}^{2}$ ) considering the same slices position. The contours of lesions were validated by another expert radiologist of 25 years of experience.

The signal intensity of the lesions in T1-w and T2-w images was categorized subjectively as isointense, hypointense, and hyperintense compared to surrounding liver parenchyma. We assessed the signal on DWI sequences and measured the apparent diffusion coefficient (ADC) of each lesion. The diffusion weighted signal decay was analyzed using the mono-exponential model, according to the equation, the apparent diffusion coefficient $\mathrm{ADC}=$ $(\ln [\mathrm{SO} / \mathrm{Sb}]) / \mathrm{b}$, where $\mathrm{Sb}$ is the signal intensity with diffusion weighting $\mathrm{b}$ and $\mathrm{S} 0$ is the non-diffusionweighted signal intensity. This analysis was ROIbased using median value of single voxel signals for each $b$ value. Median diffusion parameters of ROI were used as representative values for each lesion. No motion correction algorithm was used but ROIs were drawn taking care to exclude areas in which movement artifacts or blurring caused voxel misalignments.
We analysed the enhancement pattern during arterial, portal, equilibrium or late phase and described it as homogeneous, heterogeneous, or progressive. We described the capsule appearance, defined as a peripheral rim of smooth hyperenhancement in the portal or delayed phase, as complete or partial. In addition, we recorded the number and segmental location of the nodule for all detected lesions and the presences of satellite nodules.

\section{Statistical analyses}

Each continuous variable was expressed in terms of median value \pm range while each variable categorical was summarized by frequencies and percentages. Fisher's exact test was performed to assess statistically significant difference between percentage values. Mann Whitney non parametric test were used to compare a continuous variable between 2 groups. A p value $<0.05$ was considered statistically significant.

All statistical analysis was performed with SPSS for Windows (Version 23.0; SPSS Inc, Chicago, Ill).

\section{Results}

We assessed 62 patients that underwent surgical treatment with preoperative diagnosis of HCC. According to the surgical procedure, 10 patients underwent to lobectomy, 3 meso-hepatectomy, 23 bi-segmentectomy and 27 segmentectomy ( 8 for VII, 1 for I, 2 for II, 2 for III, 6 for IV, 6 for V and 2 for VI hepatic segment).

\section{Pathological features}

After pathological evaluation the final diagnosis was HCC for 51 patients and cHCC-CCA for 11 patients $(17.7 \%)$.

Twelve patients were classified as G3 (19.4\%) and 50 G2 (80.6\%) according to the grading system of Edmondson-Steiner. ${ }^{21}$

Among cHCC-CCA 8 patients were classified as G2 (72.7\%) and 3 as G3 (27.3\%). In 8 out of 11 $(72.7 \%)$ cHCC-CCA microvascular infiltration was reported.

In two cHCC-CCA patients were reported nodal metastases.

\section{Imaging features}

All lesions were detected and analyzed by readers. The consensus in the assessment of the nodules 


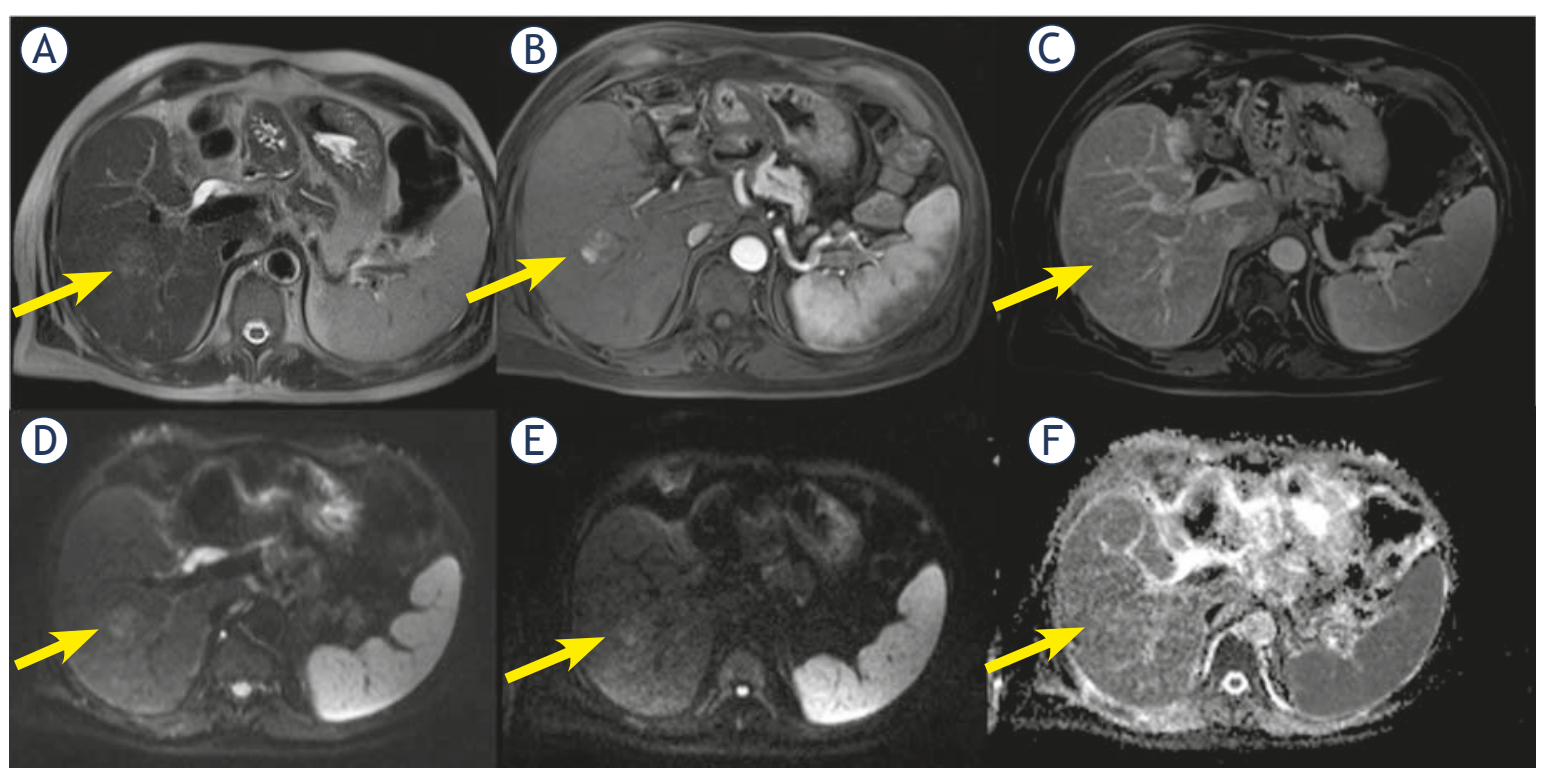

FIGURE 1. Man 56 y with combined hepatocellular-cholangiocarcinoma (cHCC-CCA) on VI hepatic segment. MRI study. The nodule is iso-hyperintense (arrow) in T2-W sequence (A), with inhomogeneous hypervascular appearance (arrow) during arterial phase of contrast study (B), without wash-out or capsule appearance (arrow) during portal phase of contrast study (C). The nodule shows restricted (arrow) diffusion (D, E and F) in diffusion weighted imaging (DWI) sequences.

was $100 \%$. The median nodule size was $46.0 \mathrm{~mm}$ (range 10-190 mm). For cHCC-CCA the median size was $33.5 \mathrm{~mm}$ (range $20-80 \mathrm{~mm}$ ), for true HCC the median size was $47.5 \mathrm{~mm}$ (range $10-190 \mathrm{~mm}$ ).

The consensus between pre-surgical radiological report and second evaluation was $100 \%$ for each LIRADS categories: LR-5 for $54(87.1 \%)$ nodules, LR-3 for 1 (1.6\%) lesion, and LR-M for 7 (11.3\%) nodules.

Thirty-nine nodules $(63 \%)$ showed hyperenhancement in arterial phase; among them 4 were cHCC-CCA (36.4\% of cHCC-CCA) and $35(68.6 \%)$ true HCC.

Twenty nodules $(32.2 \%)$ showed an inhomogeneous hyperenhancement in arterial phase; 7 of them were cHCC-CCA (63.6\% of cHCC-CCA) (Figure 1). We found this feature in $13(25.5 \%)$ true HCC.

Forty-three nodules $(69.3 \%)$ showed wash-out appearance; 6 cHCC-CCAs (54.5\% of cHCC-CCA) and 37 true HCC $(72.5 \%)$ had this feature.

We found inhomogeneous wash-out in 13 $(20.9 \%)$ nodules; 4 nodules with inhomogeneous wash-out were cHCC-CCA (35.4\% of cHCC-CCA). A one cHCC-CCA $(9.1 \%$ of cHCC-CCA) patient did not show this feature. Nine true HCC $(17.6 \%)$ showed inhomogeneous wash-out.
Thirty-three $(53.2 \%)$ nodules showed capsule appearance, $28(45.2 \%)$ did not show this feature and in one patient we found a peripheral halo sign.

Only two cHCC-CCA patients $(18.2 \%$ of cHCCCCA) showed capsule appearance while 9 cHCCCCAs ( $81.8 \%$ of cHCC-CCA) did not have this feature (Figure 2).

Thirty-one (60.8\%) true HCC showed capsule appearance and 19 (37.2\%) true HCC did not show this feature.

Only 23 patients underwent MR study, among them 7 out of cHCC-CCA. We found T2 hyperintensity of signal in 20 nodules $(86.9 \%)$, two lesions $(8.6 \%)$ were isointense and one $(4.3 \%)$ hypointense.

Five cHCC-CCA (714 \% of cHCC-CCA) showed hyperintensity on $\mathrm{T} 2-\mathrm{W}$ sequences while two $(28.6 \%)$ showed inhomogeneous signal in $\mathrm{T} 2-\mathrm{W}$ (Figure 3).

Fifteen true HCC $(93.7 \%)$ had hyperintense signal on T2-W and one true HCC (6.2\%) inhomogeneous signal on $\mathrm{T} 2-\mathrm{W}$.

We found restricted diffusion in $23(100 \%)$ nodules with median ADC of $975.6 \times 10^{-3} \mathrm{~mm}^{2} / \mathrm{s}$.

All cHCC-CCA showed restricted diffusion with median ADC of $880.7 \times 10^{-3} \mathrm{~mm}^{2} / \mathrm{s}$.

All true HCC showed restricted diffusion with median ADC of $1210.0 \times 10^{-3} \mathrm{~mm}^{2} / \mathrm{s}$. 


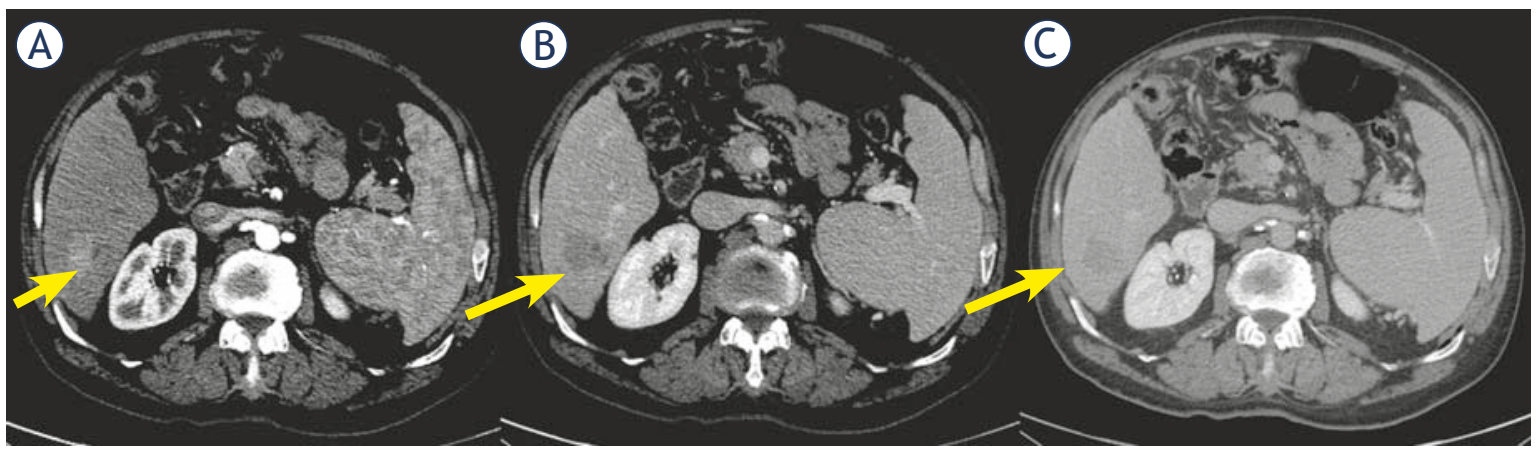

FIGURE 2. Woman 68 y with combined hepatocellular-cholangiocarcinoma (cHCC-CCA) on VI hepatic segment. Multidetector computed tomography (MDCT) study. The nodule shows hypervascular appearance (arrow) during arterial phase of contrast study (A), with wash-out appearance (arrow) and without capsule appearance (arrow) during portal and late phase of contrast study (B and C).

Progressive contrast enhancement: nine (14.5\%) patients showed a progressive contrast enhancement; among them 7 were cHCC-CCA $(63.6 \%$ of cHCC-CCA) (Figure 4$)$ and $2(3.9 \%$ of $\mathrm{HCC})$ true HCC.
In ten patients $(16.1 \%)$ we found satellite nodules (neighboring micrometastases), among them 7 were cHCC-CCA $(63.6 \%$ of cHCC-CCA) (Figure 5) and $3(5.9 \%$ of HCC $)$ were true HCC.

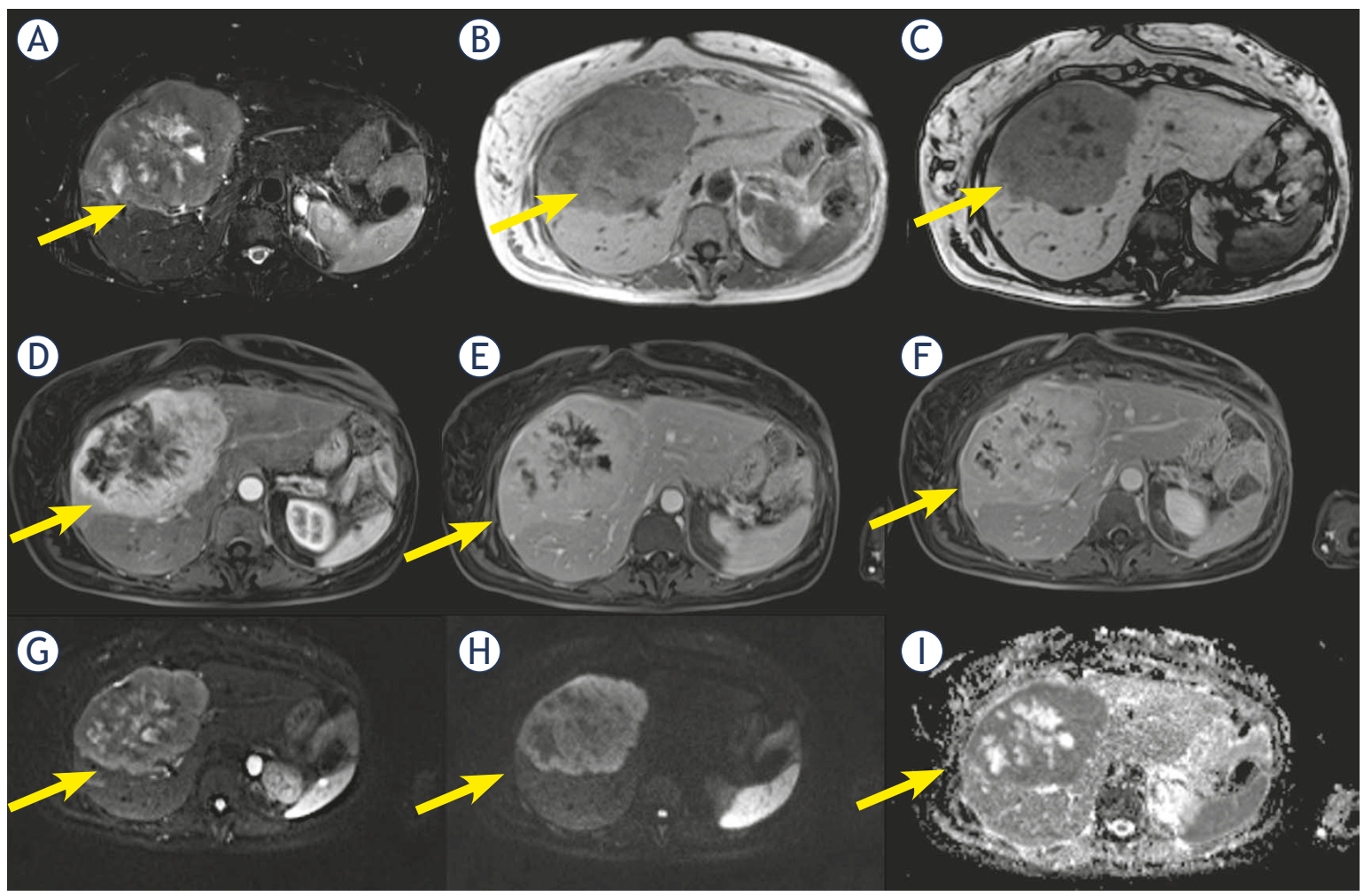

FIGURE 3. Woman 58 y with combined hepatocellular-cholangiocarcinoma (CHCC-CCA) on IV-V-VIII hepatic segment. MRI study. Pre surgical radiological diagnosis was cholangiocarcinoma (CCA). The lesion shows inhomogeneous hyperintense signal (arrow) in T2-W sequence (A) with central more hyperintense area. In T1-W in-out phase sequence (B and C) the lesion is inhomogeneous hypointense (arrow). During contrast study (D: arterial phase, E: portal phase; F: late phase) the lesion shows progressive contrast enhancement (arrow). In diffusion weighted imaging (DWI) (G, H and I) it shows restricted diffusion (arrow). 


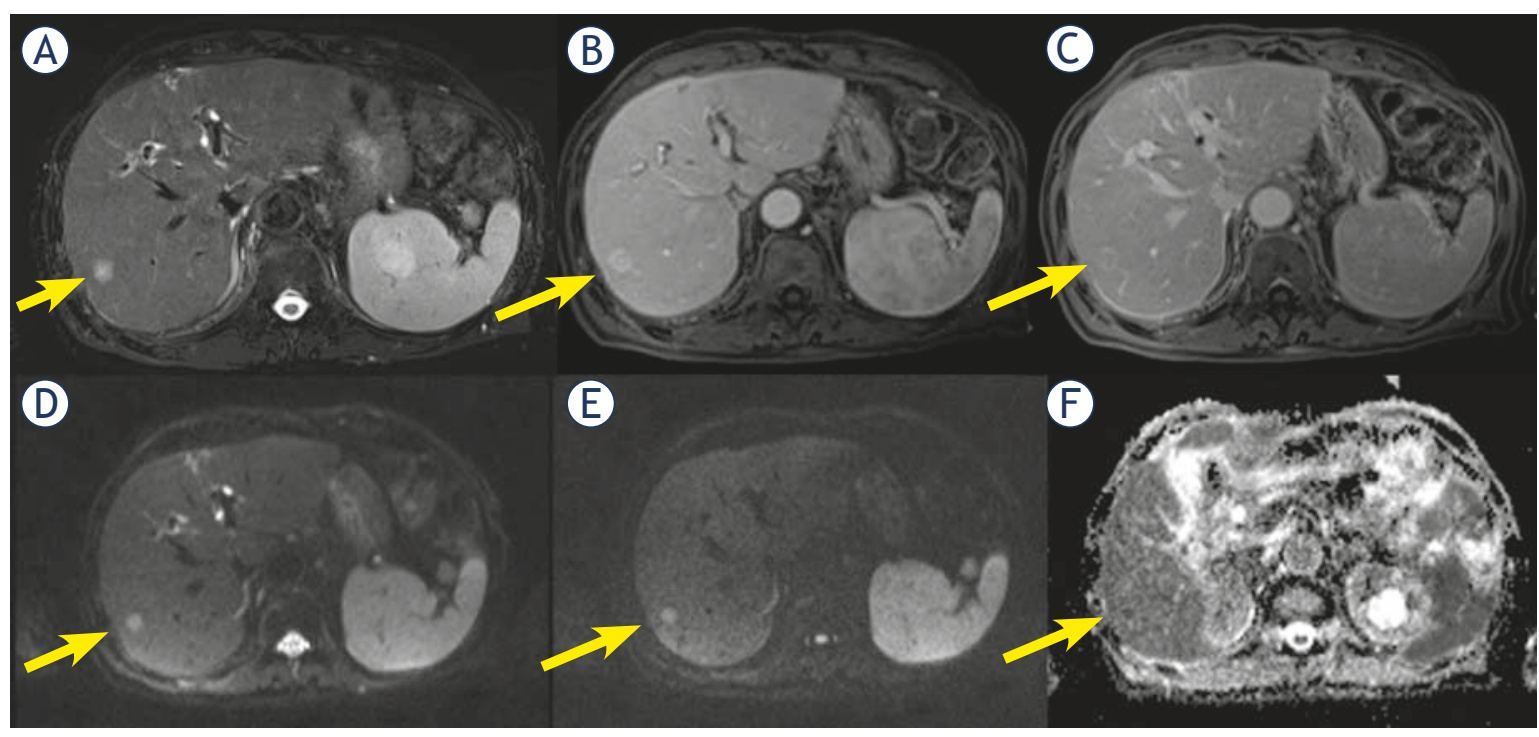

FIGURE 4. Man 71 y with combined hepatocellular-cholangiocarcinoma (CHCC-CCA) on VI hepatic segment. MRI study. The nodule shows hyperintense signal (arrow) in T2-W sequence (A) and target like pattern of enhancement (arrow) during arterial (B) and portal (C) phase of contrast study. Restricted diffusion (arrow) in diffusion weighted imaging (DWI) (D, E and F) sequence.
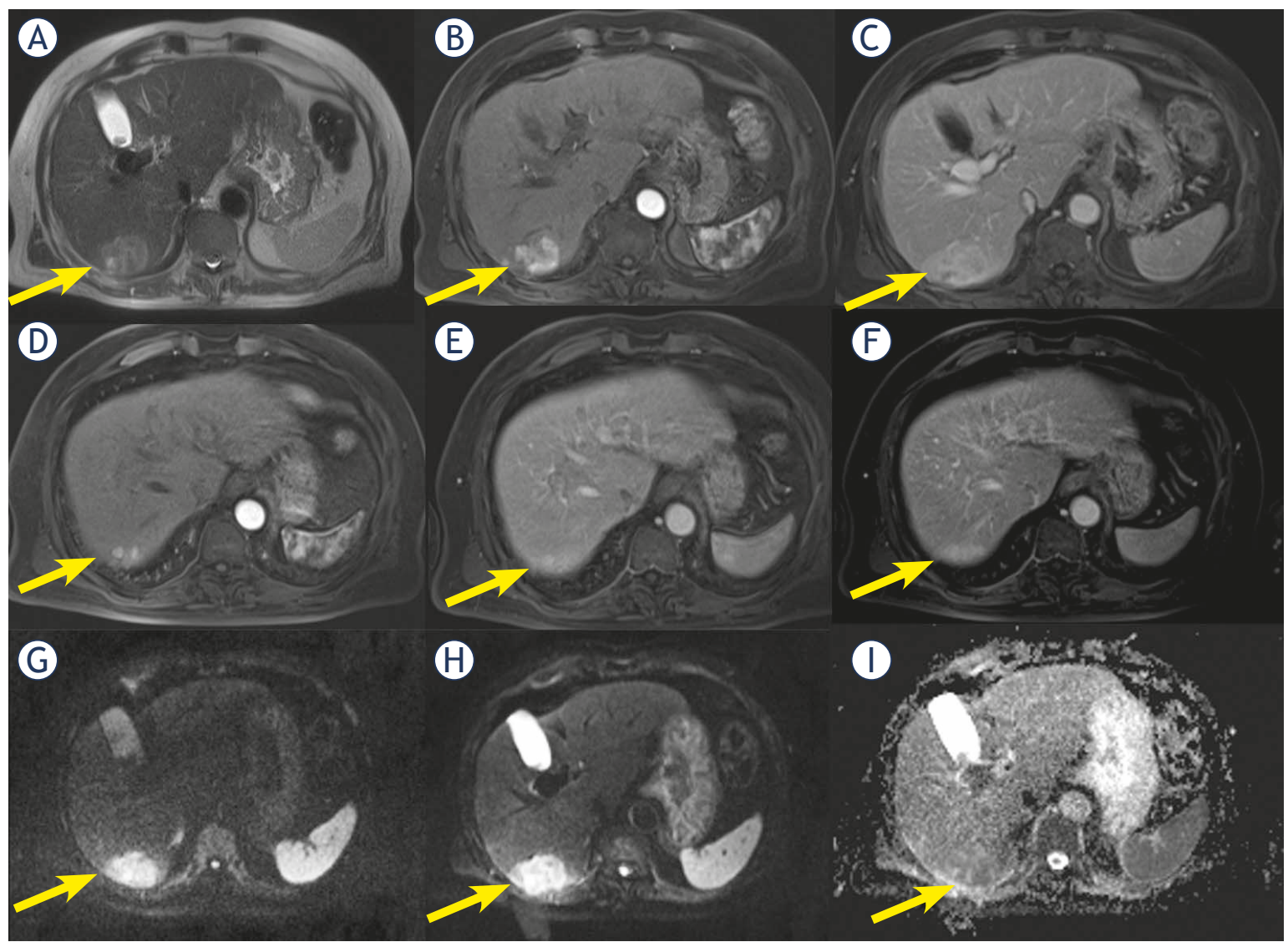

FIGURE 5. Man 69 y with combined hepatocellular-cholangiocarcinoma (cHCC-CCA) on VI hepatic segment. MRI study. The nodule shows inhomogeneous hyperintense signal (arrow) in T2-W sequence (A) and progressive pattern of enhancement (arrow) during arterial (B) and portal (C) phase of contrast study. In (D, E and F) arrow shows a nodule satellite. Restricted diffusion (arrow) in diffusion weighted imaging (DWI) (G, H and I) sequence. 
TABLE 2. Imaging features in study population

\begin{tabular}{|c|c|c|c|}
\hline & True HCC (n 51) & cHCC-CCA (n 11) & P value* \\
\hline \multicolumn{4}{|c|}{ Arterial hyperenhancement } \\
\hline Yes & $35(68.6 \%)$ & $4(36.4 \%)$ & \multirow{3}{*}{0.04} \\
\hline No & $3(5.8 \%)$ & $0(0 \%)$ & \\
\hline Inhomogeneous & $13(25.5 \%)$ & $7(63.6 \%)$ & \\
\hline \multicolumn{4}{|l|}{ Wash-out appearance } \\
\hline Yes & $37(72.5 \%)$ & $6(54.5 \%)$ & \multirow{3}{*}{0.38} \\
\hline No & $5(17.6 \%)$ & $1(9.1 \%)$ & \\
\hline Inhomogeneous & $9(17.6 \%)$ & $4(35.4 \%)$ & \\
\hline \multicolumn{4}{|l|}{ Capsule appearance } \\
\hline Yes & $31(60.8 \%)$ & $2(18.2 \%)$ & \multirow{4}{*}{0.03} \\
\hline No & $19(37.2 \%)$ & $9(81.8 \%)$ & \\
\hline Inhomogeneous & 1 (1.9\%) alo-sign & & \\
\hline MR features & 16 & 7 & \\
\hline \multicolumn{4}{|l|}{ T2-W hyperintensity } \\
\hline Yes & $15(93.7 \%)$ & $5(71.4 \%)$ & \multirow{2}{*}{0.14} \\
\hline Inhomogeneous & $1(6.2 \%)$ & $2(28.6 \%)$ & \\
\hline \multicolumn{4}{|l|}{ Diffusion restricted } \\
\hline Yes & 15 (100\%) & $7(100 \%)$ & \\
\hline No & & & \\
\hline Median ADC & $1210 \times 10^{-3} \mathrm{~mm}^{2} / \mathrm{s}$ & $880.7 \times 10^{-3} \mathrm{~mm}^{2} / \mathrm{s}$ & 0.03 \\
\hline $\begin{array}{l}\text { Progressive contrast } \\
\text { enhancement }\end{array}$ & $2(3.9 \%)$ & $7(63.6 \%)$ & $<<0.001$ \\
\hline Satellite nodules & $3(5.9 \%)$ & $7(63.6 \%)$ & $<<0.001$ \\
\hline
\end{tabular}

* Fisher's exact test

$\mathrm{ADC}=$ apparent diffusion coefficient $\mathrm{HCC}=$ hepatocellular carcinoma; $\mathrm{cHCC}-\mathrm{CCA}=\mathrm{combined}$ hepatocellular-cholangiocarcinoma; $M R=$ magnetic resonance

\section{Discussion}

In this study, we assessed 62 patients with liver single nodule that underwent surgical treatment with preoperative biopsy diagnosis of HCC and presurgical radiological diagnosis of HCC in 54 lesion and LR-M in 7 lesions.

All 7 lesions classified as LR-M were cHCCCCA; 4 cHCC-CCA patients were wrongly identified as HCC patients. In this subgroup the median size lesion was $21 \mathrm{~mm}$, while the subgroup LR-M showed a median size of $54 \mathrm{~mm}$. In this scenario we think that radiological features are also related to median lesion diameter.

When we assessed the major features, we found that arterial hyperenhancement, portal wash-out and capsule appearance were more frequent for true HCC group $(68.6 \%, 72.5 \%$, and $60.8 \%$, respectively) than cHCC-CCA group (36.4\%, 54.5\%, and $18.2 \%$, respectively). Our results are different from what is reported by Jeon et al. ${ }^{18} \mathrm{In}$ fact, these researches reported that a substantial proportion of cHCC-CCAs was categorized as LR-5 or LR-4 showing higher frequencies of major HCC features: arterial hyperenhancement was present in $96.2 \%$ of cHCC-CCA, washout appearance in $80.8 \%$ of cHCC-CCA and enhancing capsule in $34.6 \%$ of cHCC-CCA. ${ }^{18}$ Arterial phase hyperenhancement is considered a crucial precondition to define $\mathrm{HCC}^{19}$, and it is considered the most important feature for imaging diagnosis. This feature reflects the neoangiogenesis, which is associated with the stepwise process of carcinogenesis and becomes the dominant blood supply in overt HCC lesions. ${ }^{13}$ However, it is non-specific condition and may be detected in benign pathologies. Also in our previous study we demonstrated that arterial phase hyperenhancement is a prerequisite but not sufficient for LR-5 classification. ${ }^{12}$ The post-contrast feature, "rim arterial phase hyperenhancement" is a subtype of LR-M in which arterial phase enhancement is most pronounced in observation periphery and it is defined as a"target appearance". "Target appearance" or "targetoid mass" includes other features as peripheral "Washout", in which apparent washout is most pronounced in observation periphery, and delayed central enhancement, in which we find a central area of progressive post-arterial phase enhancement. In our study we found that CHCC-CCAs showed in $63.6 \%$ of lesions inhomogeneous arterial contrast enhancement, with a peripheral rim during arterial phase of contrast study and a progressive contrast enhancement ( $63.6 \%$ of lesions). Our findings 
are comparable with the results of Lee $e t$ al..$^{21}$ that found that in cHCC-CCA group the more frequent radiological findings were suggestive of LR-M. ${ }^{21}$ In fact. Also Potretzke et al. found that $93.4 \%$ of cHCC-CCA showed at least one ancillary feature favouring non-HCC malignancy. ${ }^{22}$ Conversely, Sammon et al. ${ }^{23}$ reported that arterial enhancement was seen in $90.9 \%$ (n. 30) of the cHCC-CCA group, although the most common enhancement patterns in the cHCC-CCA group were peripheral persistent and heterogeneous hyperenhancement with washout. In our study, the wash-out appearance was seen in $54.5 \%$ (n. 6) of patients, so we think it is not a feature that allow identifying that patients as a cHCC-CCA. Conversely, the absence of capsule appearance is more frequent $(81.8 \%)$ in cHCC-CCA group than in true HCC group (37.2\%). Therefore, according to our results, the absence of capsule appearance in nodule that shows peripheral and progressive contrast enhancement should guide the radiologist in differential diagnosis, since these features are more specific for $\mathrm{cHCC}$ CCA. Also Fowler et al. confirmed that peripheral arterial enhancement was the most common pattern observed in their study population. The target like or reverse target like pattern of enhancement, was a common feature of CHCC-CCA; this pattern was rarely seen with HCC in their population. ${ }^{24}$

In our study, only 23 patients were subject to pre-surgical MR study, among them only $7 \mathrm{cHCC}$ CCA patients. According to our protocol study for lesion characterization, we employed a nonhepatospecific contrast medium, so no one of our patients was subject to EOB-MR. In our previous study we showed that when we analyzed the degree of arterial phase hyperenhancement, we found that the degree was higher with Gd-BT-DO3A than GD-EOB-DTPA, with significant statistically difference. In addition, the image quality degradation was lower with Gd-BT- DO3A than with GD-EOBDTPA. There was significant statistically difference between the quality on arterial phase with Gd-BTDO3A and the quality on arterial phase with GDEOB-DTPA. ${ }^{12}$

Regarding the signal observed on $\mathrm{T} 2-\mathrm{W}$ sequences we found that $15 / 16$ (93.7\%) of true HCC showed hyperintense signal on $\mathrm{T} 2-\mathrm{W}$ and $5 / 7(71.4 \%)$ of cHCC-CCA had this feature. T2-W hyperintensity was a highly specific marker of nodule malignancy, although poorly sensitive. ${ }^{13}$ Kim et al..$^{25}$ evaluated the most predictive finding among hyperintensity on T2-W, DWI, washout, capsular enhancement, and hypointensity on gadoxetic acid-enhanced hepatobiliary phase images in the detailed char- acterization of arterial phase enhancing nodules 1 $\mathrm{cm}$ in diameter and smaller. They showed that for hypervascular lesions $1 \mathrm{~cm}$ in diameter or smaller, T2-weighted images have the highest sensitivity among tests with an odds ratio statistically separable from 1 for differentiating HCC from benign hypervascular lesions $1 \mathrm{~cm}$ or smaller. ${ }^{25}$ Our results confirmed that hypeintensity on $\mathrm{T} 2-\mathrm{W}$ is suggestive of malignancy, in fact 20/23 nodules $(86.9 \%)$ showed this feature, however it is not allow characterizing CHCC-CCA as a separate lesion to HCC.

In our study, all cHCC-CCA patients showed restricted diffusion with a median ADC value of 880.7 $x 10^{-3} \mathrm{~mm}^{2} / \mathrm{s}$ so as all true HCC showed restricted diffusion with median ADC of $1210.0 \times 10^{-3} \mathrm{~mm}^{2} / \mathrm{s}$. DWI has been applied to liver imaging as an excellent tool for detection and characterization of focal liver lesions, increasing clinical confidence and decreasing false positives. ${ }^{11}$ DWI could be used as a helpful diagnostic tool for HCC in patients with chronic liver disease, since DWI can accurately detect HCC in patients with chronic liver disease regardless of the lesion size. ${ }^{11,12} \mathrm{~A}$ potential reason for the better accuracy of DWI is that this does not rely on morphologic features only. Malignant tissues tend to be hypercellular with an accumulation of macromolecular proteins leaving a small extracellular space resulting in a decrease of the ADC value. The major limits of DWI are the different parameters used in DWI sequences that may affect the results of ADC calculation. ${ }^{11,12}$ In our study, ADC value could allow to distinguish cHCC-CCA as a separate group from true HCC patients.

An interesting radiological finding that we found was the presence in cHCC-CCA group of satellite nodules, expression of micrometastases, as confirmed by pathologist. In fact, we found this finding in $63.6 \%$ (n. 7) cHCC-CCA patients and only in $5.9 \%$ (n. 3) true HCC. At the best of our knowledge, no previous study reported this result. We think that it is linked to more aggressiveness of this type of lesion, data confirmed also by the presence of nodal metastases in two cHCC-CCA patients.

There are several limitations to our study. First, the sample size was small because of the rarity of cHCC-CCA and it is a single-center experience. Second that this is a retrospective study and the readers were aware, the cohort comprised of cHCC-CC and HCC. Third, the study is defective of radiology-pathology correlation. Our future prospective is to assess radiology-pathology correlation to better show how the relative composition of these mixed tumours translates into their imaging appearance. 


\section{Conclusions}

A proper diagnosis of cHCC-CCA is mandatory given that surgical resection is the current standard of care for cHCC-CCAs and controversies surround the appropriateness of other therapies. An exact pre-surgical diagnosis is very complicated due to its heterogeneous imaging characteristics with overlapping features of HCC and CCA. However according to our results in patients at risk for HCC, when the lesion shows satellite nodules, hyperintense signal on $\mathrm{T} 2-\mathrm{W}$, restricted diffusion and especially the absence of capsule appearance in nodule that shows peripheral and progressive contrast enhancement, the radiologist should consider the diagnosis of cHCC-CCA.

\section{Acknowledgements}

The authors are grateful to Alessandra Trocino, librarian at the National Cancer Institute of Naples, Italy. Moreover, for the collaboration, authors are grateful to Assunta Zazzaro and Dr Ivano Rossi, TSRM at Radiology Division, "Istituto Nazionale Tumori IRCCS Fondazione Pascale - IRCCS di Napoli", Naples, I-80131, Italy.

\section{References}

1. Garancini M, Goffredo P, Pagni F, Romano F, Roman S, Sosa JA, et al. Combined hepatocellular-cholangiocarcinoma: a population-level analysis of an uncommon primary liver tumor. Liver Transpl 2014; 20: 952-9. doi: $10.1002 /$ lt.23897

2. Joo I, Kim H, Lee JM. Cancer stem cells in primary liver cancers: pathological concepts and imaging findings. Korean J Radiol 2015; 16: 50-68. doi: 10.3348/kjr.2015.16.1.50

3. Gera S, Ettel M, Acosta-Gonzalez G, Xu R. Clinical features, histology, and histogenesis of combined hepatocellular-cholangiocarcinoma. World J Hepato 2017; 9: 300-9. doi: 10.4254/ wjh. v9. i6.300

4. Akiba J, Nakashima O, Hattori S, Tanikawa K, Takenaka M, Nakayama M, et al. Clinicopathologic analysis of combined hepatocellular-cholangiocarcinoma according to the latest WHO classification. Am J Surg Pathol 2013; 37: 496-505. doi: 10.1097/PAS.0b013e31827332b0

5. Portolani N, Baiocchi GL, Coniglio A, Piardi T, Grazioli L, Benetti A, et al. Intrahepatic cholangiocarcinoma and combined hepatocellular-cholangiocarcinoma: a Western experience. Ann Surg Oncol 2008; 15: 1880-90. doi: 10.1245/s10434-008-9933-y

6. Yeh MM. Pathology of combined hepatocellular-cholangiocarcinoma. J Gastroenterol Hepatol 2010; 25: 1485-92. doi: 10.1111/j.1440-1746. $2010.06430 x$

7. Yoon YI, Hwang S, Lee YJ, Kim KH, Ahn CS, Moon DB, et al. Postresection outcomes of combined hepatocellular carcinoma-cholangiocarcinoma, hepatocellular carcinoma and intrahepatic cholangiocarcinoma. J Gastrointest Surg 2016; 20: 411-20. doi: 10.1007/s11605-015-3045-3

8. Allen RA, Lisa JR. Combined liver cell and bile duct carcinoma. Am J Pathol 1949; 25: 647-55. PMID: 18152860
9. Okuda K. Natural history of hepatocellular carcinoma including fibrolamellar and hepato-cholangiocarcinoma variants. J Gastroenterol Hepatol 2002; 17: 401-5. doi: 10.1046/j.1440-1746.2002.02734.x

10. Zhou YM, Zhang XF, Wu LP, Sui CJ, Yang JM. Risk factors for combined hepatocellular-cholangiocarcinoma: a hospital-based case-control study. World J Gastroenterol 2014; 20: 12615-20. doi: 10.3748/wjg.v20.i35.12615

11. Granata V, Fusco R, Filice S, Catalano O, Piccirillo M, Palaia R, et al. The current role and future prospectives of functional parameters by diffusion weighted imaging in the assessment of histologic grade of HCC. Infect Agent Cancer 2018; 13: 23. doi: 10.1186/s13027-018-0194-5

12. Granata V, Fusco R, Avallone A, Filice F, Tatangelo F, Piccirillo M, et al. Critical analysis of the major and ancillary imaging features of LI-RADS on 127 proven HCCs evaluated with functional and morphological MRI: lights and shadows. Oncotarget 2017; 8: 51224-37. doi: 10.18632/oncotarget.17227

13. Granata V, Fusco R, Avallone A, Catalano O, Filice F, Leongito M, et al. Major and ancillary magnetic resonance features of LI-RADS to assess HCC: an overview and update. Infect Agent Cancer 2017; 12: 23. doi: 10.1186/ s13027-017-0132-y

14. Granata V, Fusco R, Catalano O, Guarino B, Granata F, Tatangelo F, et al. Intravoxel incoherent motion (IVIM) in diffusion-weighted imaging (DWI) for hepatocellular carcinoma: correlation with histologic grade. Oncotarget 2016; 7: 79357-64. doi: 10.18632/oncotarget.12689

15. Granata V, de Lutio di Castelguidone E, Fusco R, Catalano O, Piccirillo M, Palaia R, et al. Irreversible electroporation of hepatocellular carcinoma: preliminary report on the diagnostic accuracy of magnetic resonance, computer tomography, and contrast-enhanced ultrasound in evaluation of the ablated area. Radiol Med 2016; 121: 122-31. doi: 10.1007/s11547015-0582-5.

16. Zhang $X Y$, Luo $Y$, Wen TF, Jiang $L$, Li $C$, Zhong $X F$, et al. Contrast-enhanced ultrasound: improving the preoperative staging of hepatocellular carcinoma and guiding individual treatment. World J Gastroenterol 2014; 20: 1262836. doi: $10.3748 /$ wjg.v20.i35.12628

17. Ayuso C, Rimola J, García-Criado A. Imaging of HCC. Abdom Imaging 2012 37: 215-30. doi: 10.1007/s00261-011-9794-x

18. Jeon SK, Joo I, Lee DH, Lee SM, Kang HJ, Lee KB, et al. Combined hepatocellular cholangiocarcinoma: LI-RADS v2017 categorisation for differentia diagnosis and prognostication on gadoxetic acid-enhanced MR imaging. Eur Radiol 2019; 29: 373-82. doi: 10.1007/s00330-018-5605-x

19. The American College of Radiology. Liver Reporting \& Data System (LIRADS). [cited 2020 Jan 15]. Available at: https://www.acr.org/ClinicalResources/Reporting-and-Data-Systems/LI-RADS

20. Edmondson HA, Steiner PE. Primary carcinoma of the liver: a study of 100 cases among 48,900 necropsies. Cancer 1954; 7: 462-503. doi: 10.1002/1097-0142(195405)7:3<462::aid-cncr2820070308>3.0.co;2-e

21. Lee HS, Kim MJ, An C. How to utilize LR-M features of the LI-RADS to improve the diagnosis of combined hepatocellular-cholangiocarcinoma on gadoxetate-enhanced MRI? Eur Radiol 2019; 29: 2408-16. doi: 10.1007/ s00330-018-5893-1

22. Potretzke TA, Tan BR, Doyle MB, Brunt EM, Heiken JP, Fowler KJ. Imaging features of biphenotypic primary liver carcinoma (hepatocholangiocarcinoma) and the potential to mimic hepato- cellular carcinoma: LI-RADS analysis of CT and MRI features in 61 cases. AJR Am J Roentgenol 2016; 207: 25-31. doi: 10.2214/AJR.15.14997

23. Sammon J, Fischer S, Menezes R, Hosseini-Nik H, Lewis S, Taouli B, et al. MRI features of combined hepatocellular-cholangiocarcinoma versus mass forming intrahepatic cholangiocarcinoma. Cancer Imaging 2018; 18: 8. doi: 10.1186/s40644-018-0142-z

24. Fowler KJ, Sheybani A, Parker RA 3rd, Doherty S, M Brunt E, Chapman WC, et al. Combined hepatocellular and cholangiocarcinoma (biphenotypic) tumors: imaging features and diagnostic accuracy of contrast-enhanced CT and MRI. AJR Am J Roentgenol 2013; 201: 332-9. doi: 10.2214/AJR.12.9488

25. Kim JE, Kim SH, Lee SJ, Rhim H. Hypervascular hepatocellular carcinoma 1 $\mathrm{cm}$ or smaller in patients with chronic liver disease: characterization with gadoxetic acid-enhanced MRI that includes diffusion-weighted imaging. AJR Am J Roentgenol 2011; 196: W758-65. doi: 10.2214/AJR.10.4394 